\title{
Autosomal recessive congenital cerebellar ataxia due to MGLUR1 deficiency
}

INSERM

\section{Source}

INSERM. (1999). Orphanet: an online rare disease and orphan drug data base. Autosomal recessive congenital cerebellar ataxia due to MGLUR1 deficiency. ORPHA:324262

Autosomal recessive congenital cerebellar ataxia due to MGLUR1 deficiency is a rare, genetic, slowly prog ressive neurodegenerative disease resulting from MGLUR1 deficiency characterized by global developmental delay (beginning in infancy), mild to severe intellectual deficit with poor or absent speech, moderate to severe stance and gait ataxia, pyramidal signs (e.g. hyperreflexia) and mild dysdiadochokinesia, dysmetria, tremors, and/or dysarthria. Oculomotor signs, such as nystagmus, strabismus, ptosis and hypometric saccades, may also be associated. Brain imaging reveals progressive, generalized, moderate to severe cerebellar atrophy, inferior vermian hypoplasia, and/or constitutionally small brain. 